\title{
A Comparison of Matrix-Assisted Laser Desorption/lonization Time-of-Flight Mass Spectrometry and Surface Plasmon Resonance for Genotyping of High-Risk Human Papillomaviruses
}

\author{
Shoufang $\mathrm{Qu}^{\mathrm{a}, \mathrm{c}}$ Jie Huang ${ }^{\mathrm{c}}$ Jinyin Zhao ${ }^{d}$ Xuequan Zhao ${ }^{\mathrm{b}}$ Haijun Deng ${ }^{\mathrm{d}}$ \\ Huawei Yang ${ }^{e}$ Weijun Chen ${ }^{d}$ Licheng Liu ${ }^{d, f}$ Lining Zhang ${ }^{a}$ Shangxian Gao ${ }^{a, c}$ \\ ${ }^{a}$ Department of Immunology, Shandong University School of Medicine, and ${ }^{\mathrm{b} C h i n a}$ Unicom System Integration \\ Limited Corporation Shandong Branch, Ji'nan, and 'Department of in vitro Diagnostic Reagents and Medium, \\ National Institute for the Control of Pharmaceutical and Biological Products, ${ }^{d}$ Beijing Institute of Genomics, \\ Chinese Academy of Sciences, ${ }^{e}$ Beijing GP Medical Technologies Ltd., and ${ }^{\mathrm{f}}$ Beijing Genomics Institute in Beijing, \\ Beijing, China
}

\section{Key Words}

Genotype · Human papillomavirus · Matrix-assisted laser desorption/ionization time-of-flight mass spectrometry • Surface plasmon resonance $\cdot$ Time-of-flight mass spectrometry

\begin{abstract}
Background: Human papillomavirus (HPV) testing coupled with appropriate clinical management is associated with a significant decline in the rate of advanced cervical cancer and associated death. Methods: In this present study, we evaluated the performance of 2 new HPV genotyping methods, matrix-assisted laser desorption/ionization time-offlight mass spectrometry (MALDI-TOFMS) and surface plasmon resonance (SPR) in 30 kinds of HPV control materials and in 129 cases of cervical smears including 79 HPV-positive samples screened from 1,600 abnormal clinical samples and 50 cervical cytology samples. Results: The HPV genotyping accuracy of both MALDI-TOFMS and SPR was $100 \%$ for the HPV genotyping of control materials. In the analysis of the 79 HPV-positive samples by MALDI-TOFMS, HPV positivity was 88.6\% (70/79). Nine samples were non-high-risk HPV (nonHR-HPV), which were not targets of MALDI-TOFMS. In the
\end{abstract}

analysis of the 50 cervical samples, the agreement of both tests was $84 \%$ with a $\kappa$ value of 0.660 . By using consensus results that mean agreement between 2 of 3 methods, the HR-HPV genotyping accuracy was $100 \%$ (77/77) by MALDITOFMS and $94.8 \%(73 / 77)$ by SPR in the 129 cervical samples. The sensitivity $(88.2 \% ; 82 / 93)$ and specificity $(88.9 \% ; 32 / 36)$ of MALDI-TOFMS were similar to those of SPR. Conclusion: These results support that MALDI-TOFMS is a sensitive, specific and feasible method for HR-HPV detection in clinical application, compared with the SPR method.

Copyright $\odot 2011$ S. Karger AG, Basel

\section{Introduction}

Cervical cancer is the second most common cancer in women worldwide. Epidemiologic studies showed that infections with high-risk human papillomaviruses (HRHPV) were responsible for the development of cervical cancer and cervical intraepithelial neoplasias [1-3]. Sankaranarayanan et al. [4] found that HR-HPV testing coupled

S.Q., J.H. and J.Z. contributed equally to this study.

\section{KARGER}

Fax +4161306 1234

E-Mail karger@karger.ch

www.karger.com
(C) 2011 S. Karger AG, Basel

$0300-5526 / 11 / 0546-0326 \$ 38.00 / 0$

Accessible online at:

www.karger.com/int
Shangxian Gao, MD, PhD

Department of In Vitro Diagnostic Reagents and Medium

National Institute for the Control of Pharmaceutical and Biological Products

No. 2 Tiantan Xi Li, Beijing 100050 (China)

Tel. +8610 6709 5647, Fax +86106702 0119, E-Mail gaoshangxian@yahoo.cn 
with appropriate clinical management associate with a significant decline in the rate of advanced cervical cancer and associated death. Indeed, HR-HPV DNA could be detected in almost all cervical cancer patients [5]. Thus, HRHPV DNA detection in cervical smears could be of value in the prevention and diagnosis of cervical cancer [6].

Today, more than 120 types of HPV have been recognized on the basis of DNA sequence data. Based on their association with cervical cancer and precursor lesions, HPV has been grouped into HR-HPV and low-risk HPV (LR-HPV) types [7]. HR-HPV mainly include types 16, $18,31,33,35,39,45,51,52,56,58,59$ and 68; LR-HPV mainly include types $6,11,40,42-44,54,70$ and 81 . Several studies have shown that different HPV types play individual roles in cervical cancer development and precursor lesions. For example, prevalent infection with HPV16 is associated with a 5 times greater risk of cervical intraepithelial neoplasia 3 than infection with other prevalent oncogenic HPV types, and causes more than $50 \%$ of cervical cancer $[2,8]$, while HPV 18 causes $10-15 \%$ of cervical cancer and more than $35 \%$ of cervical adenocarcinomas [2]. Following HPV16 and 18, HPV45, 31 and other types are also important. Moreover, multiple HPV infections increase the risk of cervical cancer $[9,10]$. These findings have indicated that HPV genotyping could play an important role in the management of cervical cancer and prediction of the outcome of HPV infection.

During the last 10 years, hybrid capture 2 (HC2) has been the major means for HPV genotyping [11]. However, it does not identify specific HPV types or quantitate the HPV [12]. Furthermore, HC2 is known to have a significant rate of false-positive reactions owing to cross-reactivity with LR-HPV types (approx. 10-19\%) [13-16]. Other drawbacks to the HC2 test are its cost (USD 20-30 per test) and that it is time-consuming. Recently, new methods such as surface plasmon resonance (SPR) and matrixassisted laser desorption/ionization time-of-flight mass spectrometry (MALDI-TOFMS) have reported high sensitivity and convenience $[12,17-20]$. To evaluate the efficiency of these two methods, we were interested in comparing the results from 129 cervical samples by the MALDI-TOFMS and SPR technologies for the detection of HR-HPV genotypes.

\section{Materials and Methods}

\section{Patients and Samples}

The 30 control samples containing HR-HPV genotypes 16, 18, $31,33,35,39,45,51-53,56,58,59,66,68$ and 73 as well as LR-HPV genotypes $6,11,26,40,42-44,54,61,70,72,81,83$ and CP8304 were provided by the National Institute for the Control of Pharmaceutical and Biological Products (Beijing, China). The original concentration of the 30 control samples was from $10^{8}$ to $10^{9}$ copies per milliliter. Then the diluted control samples (from $10^{5}$ to $10^{6}$ copies $/ \mathrm{ml}$ ) were used as a template in the experiment. Seventynine cervical samples having pathological changes more extensive than atypical squamous cells (ASC) of undetermined significance were identified from 1,600 cervical smears obtained from outpatients with abnormal cytology from June 2007 to June 2008. These had been found HPV positive by SPR. Another 50 cervical samples collected from outpatients in Beijing Women's Hospital and Beijing People's Hospital from July 2008 to August 2008 were graded based on The Bethesda System for Reporting Cervical Cytology (USA). They were divided into negative for intraepithelial lesion and malignancy (NILM; $\mathrm{n}=40$ ), atypical squamous cells (ASC; $\mathrm{n}=3$ ) - which includes ASC of undetermined significance and ASC-cannot exclude high-grade squamous intraepithelial lesion (HSIL) -, low-grade squamous intraepithelial lesion (LSIL; $\mathrm{n}=5)$, HSIL $(\mathrm{n}=1)$ and squamous cell carcinoma (SCC; $\mathrm{n}=1$ ). All the cervical samples were obtained from patients by using the Rovers Cervex-Brush (Therapak, Oss, The Netherlands). All samples were aliquoted and stored frozen at $-20^{\circ}$. Cellular and viral DNA was extracted by using the QIAamp DNA Mini Kit (Qiagen) according to the 'blood and body fluid spin protocol'.

\section{MALDI-TOFMS Test}

The MALDI-TOFMS test is based on PCR amplification and single-base extension (BGI Corp., Shenzhen, China). Briefly, according to the manufacturer's instructions, the consensus primers (GP6/GP5 primers: TTTGTTACTGTGGTAGATACTAC/ GAAAAATAAACTGTAAATCATATTC) were used to amplify 14 HR-HPV $(16,18,31,33,35,39,45,51,52,56,58,59,66,68)$. A thermocycler (Bio-Rad, Hercules, Calif., USA) was used, the initial heating step was at $95^{\circ}$ for $5 \mathrm{~min}$, followed by 35 cycles of $95^{\circ}$ for $15 \mathrm{~s}, 55^{\circ}$ for $15 \mathrm{~s}$, and $72^{\circ}$ for $30 \mathrm{~s}$. Single-base extension primers are specified for each HR-HPV and can be differentiated by MALDI-TOFMS. The analysis is performed at $94^{\circ}$ for $30 \mathrm{~s}$, primer annealing at $53^{\circ}$ for $40 \mathrm{~s}$, and primer extension at $72^{\circ}$ for $40 \mathrm{~s}$, 50 cycles, and finally at $72^{\circ}$ for $3 \mathrm{~min}$. The $\beta$-globin housekeeping gene was used as performance and integrity control. Controls for MALDI-TOFMS included: no template control (water), human control DNA and HPV18 plasmid DNA.

Sample aliquots were deposited onto AnchorChip ${ }^{\mathrm{TM}}$ (Bruker Daltonik GmbH, Leipzig, Germany) $400-\mu \mathrm{m}$ targets prespotted with matrix. The matrix employed was a saturated solution of 3-hydroxypicolinic acid (Sigma-Aldrich Chemie $\mathrm{GmbH}$, Buchs, Switzerland) in acetonitrile/water 1:1 (Merck, Germany) mixed with $0.4 \mathrm{M}$ dibasic ammonium citrate (Sigma-Aldrich) at a 9:1 volume ratio. All the solvents utilized were of analytical grade or at least specified for mass spectrometry. Mass spectra were recorded on a Reflex IV MALDI-TOFMS (Bruker Daltonik) that was operated in the linear positive ion mode. A 337-nm nitrogen laser with a $9-\mathrm{Hz}$ pulse frequency was used. Mass spectrometric parameters were optimized for the $\mathrm{m} / \mathrm{z}$ range from 1,000 to 10,000 , using the mass spectra of peptides for calibration. For each mass spectrum, 30 laser pulses were summed at a constant laser power and constant threshold value in order to enhance the resolution. Registration and analysis of spectra were executed using XMASS and FlexAnalysis version 2.4 (Bruker Daltonik). Three times, independent analyses were run to test the detection level. 
Table 1. Results of HPV genotyping of control materials by SPR and MALDI-TOFMS

\begin{tabular}{|c|c|c|c|}
\hline \multirow{2}{*}{$\begin{array}{l}\text { Specimen } \\
\text { No. }\end{array}$} & \multicolumn{3}{|c|}{ Genotyping, type } \\
\hline & sequencing & SPR & MALDI-TOFMS \\
\hline \multicolumn{4}{|l|}{ HR-HPV } \\
\hline 1 & 16 & 16 & 16 \\
\hline 2 & 18 & 18 & 18 \\
\hline 3 & 31 & 31 & 31 \\
\hline 4 & 33 & 33 & 33 \\
\hline 5 & 35 & 35 & 35 \\
\hline 6 & 39 & 39 & 39 \\
\hline 7 & 45 & 45 & 45 \\
\hline 8 & 51 & 51 & 51 \\
\hline 9 & 52 & 52 & 52 \\
\hline 10 & 53 & 53 & - \\
\hline 11 & 56 & 56 & 56 \\
\hline 12 & 58 & 58 & 58 \\
\hline 13 & 59 & 59 & 59 \\
\hline 14 & 66 & 66 & 66 \\
\hline 15 & 68 & 68 & 68 \\
\hline 16 & 73 & - & - \\
\hline \multicolumn{4}{|l|}{ LR-HPV } \\
\hline 1 & 6 & 6 & - \\
\hline 2 & 11 & 11 & - \\
\hline 3 & 26 & - & - \\
\hline 4 & 40 & 40 & - \\
\hline 5 & 42 & 42 & - \\
\hline 6 & 43 & 43 & - \\
\hline 7 & 44 & 44 & - \\
\hline 8 & 54 & 54 & - \\
\hline 9 & 61 & - & - \\
\hline 10 & 70 & 70 & - \\
\hline 11 & 72 & - & - \\
\hline 12 & 81 & 81 & - \\
\hline 13 & 83 & - & - \\
\hline 14 & CP8304 & CP8304 & - \\
\hline
\end{tabular}

SPR Test

SPR is a PCR-based method for the genotyping of HPV DNA from HR-HPV $(16,18,31,33,35,39,45,51-53,56,58,59,66,68$, $81)$ and LR-HPV types $(6,11,40,42-44,54,70)$. Cervical samples were tested according to the manufacturer's instructions (GP Medical Technologies, Beijing, China). Briefly, cervical sample DNA was amplified by consensus primers (provided by the kit), performed at $50^{\circ}$ for $2 \mathrm{~min}$ and $95^{\circ}$ for $4 \mathrm{~min}$, followed by $94^{\circ}$ for $30 \mathrm{~s}, 48^{\circ}$ for $45 \mathrm{~s}$ and $72^{\circ}$ for $20 \mathrm{~s}\left(28\right.$ cycles), and $94^{\circ}$ for $30 \mathrm{~s}, 65^{\circ}$ for $45 \mathrm{~s}$ and $72^{\circ}$ for $20 \mathrm{~s} \mathrm{(} 25 \mathrm{cycles}$ ), at $4^{\circ}$ hold. PCR products were denatured at $95^{\circ}$ for $5 \mathrm{~min}$, followed by $1 \mathrm{~min}$ on ice. The hybridization measurement was conducted by injecting $100 \mu \mathrm{l}$ of PCR product at a flow rate of $5 \mu \mathrm{l} / \mathrm{min}$. The reaction was monitored for $15 \mathrm{~min}$, and then the sensor chip W2600 (GP Medical Technologies) was automatically washed with hybridization buffer to remove the unbound DNA material. The analytical signal, reported as resonance units, was derived by the difference between the fi- nal value and the value recorded before the hybridization. These analyses were repeated 3 times.

\section{Sequencing}

Samples with discordant results between SPR and MALDITOFMS were resolved by sequencing. Each discordant sample was amplified by MY11 and MY09 primers [21]. Sequencing was performed by the Sangon Corp. (Beijing, China) using the ABI PRISM 3730xl system (Applied Biosystems, USA).

\section{Statistical Analysis}

A significance level of 0.05 was used in comparing performance characteristics. Agreement between pairs of tests was assessed using Cohen's $\kappa$, with values in the range of $0.00-0.20$ indicating poor agreement, $0.21-0.40$ fair agreement, $0.41-0.60$ moderate agreement, $0.61-0.80$ good agreement, and $0.81-1.00$ excellent agreement. The genotyping accuracy of HR-HPV detection was calculated as the percentage of correct results by the respective HPV tests compared to consensus HR-HPV results (defined as 2 out of 3 positive or negative HPV test results).

\section{Results}

Accuracy of MALDI-TOFMS and SPR for HR-HPV Genotyping in HPV Control Materials Containing 30 Genotypes

Analytical results of the reference control samples containing 16 documented HR-HPV and 14 LR-HPV genotypes were used to evaluate the accuracy of the SPR and MALDI-TOFMS methods. As shown in table 1, MALDI-TOFMS as well as SPR correctly identified all HR-HPV genotypes. Furthermore, the results for LRHPV were correct only for the specific low-risk genotypes targeted by the method of SPR. These demonstrate that the accuracy of MALDI-TOFMS for HR-HPV genotyping reaches $100 \%$, as well as that of SPR, suggesting that both MALDI-TOFMS and SPR are reliable for HR-HPV genotyping.

Application of MALDI-TOFMS and SPR for HR-HPV Genotyping to 129 Clinical Cervical Samples

To test whether MALDI-TOFMS can identify HRHPV types well in clinical samples, we used the MALDITOFMS method to analyze $79 \mathrm{HPV}$-positive samples screened by SPR from 1,600 abnormal cervical samples. One representative result detected each by MALDITOFMS and SPR is shown in figure 1 . As shown in table 2, MALDI-TOFMS could successfully genotype 70 of 79 samples $(88.6 \%)$. The concordance between MALDITOFMS and SPR was $88.6 \%$ (62/70). As shown in table 3, 8 cases of discordance between MALDI-TOFMS and SPR were further retested by sequencing to ascertain a con- 
Fig. 1. Results of SPR (a) and MALDITOFMS (b) analyses of 1 HPV16-positive sample. a HPV16 produced a positive signal, while the other types were all negative. b Mass spectrum for UEP-HPV16, the HPV16 unextended extension primers, and HPV16, the HPV16 extension product. The HPV16 spectrum had a positive result.

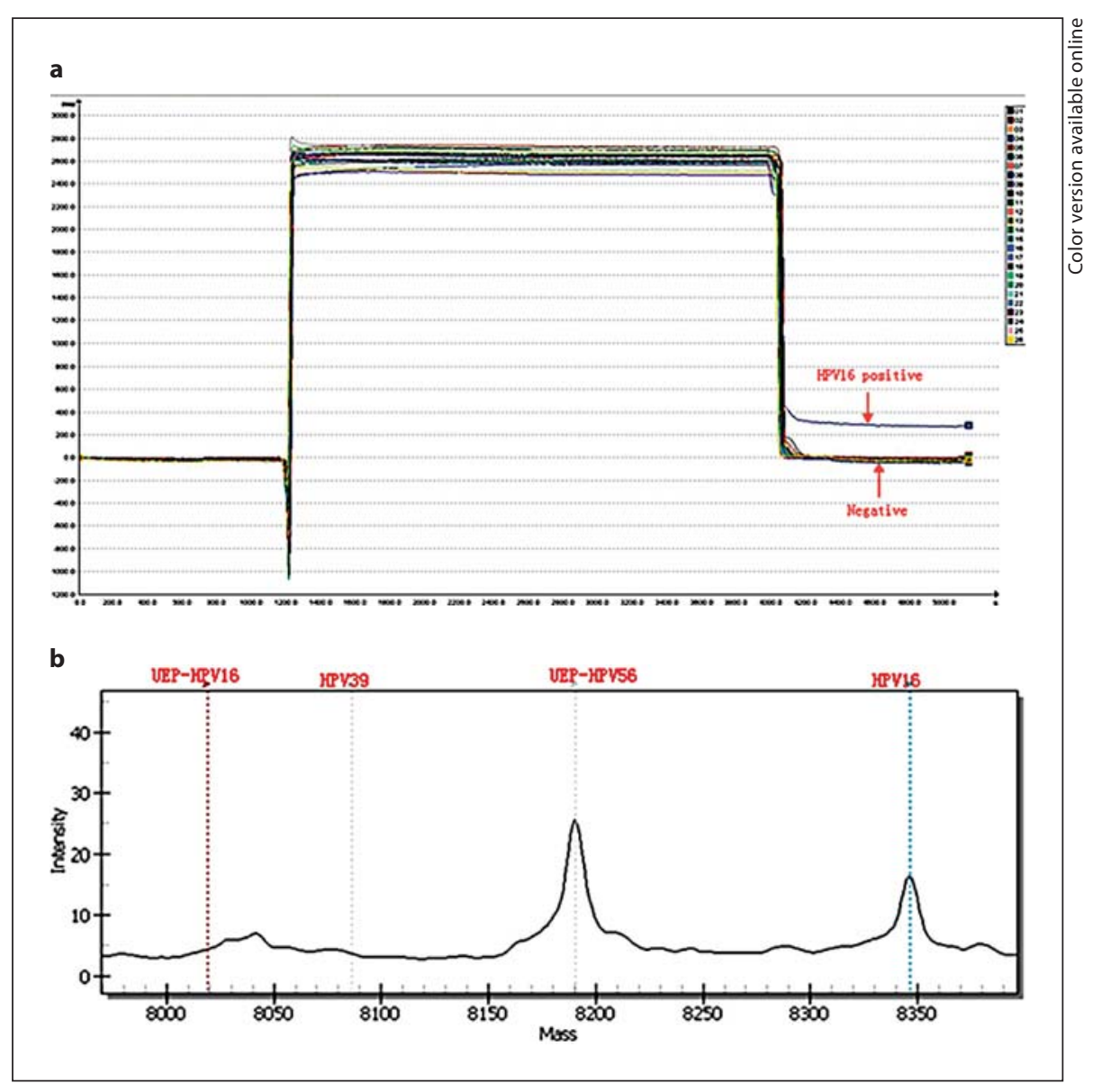

sensus result (defined as 2 out of 3 positive or negative HPV test results). Thus, there were $65 \mathrm{HR}-\mathrm{HPV}$ and 14 LR-HPV among the 79 samples. The HR-HPV genotyping accuracy was $100 \%(65 / 65)$ by MALDI-TOFMS and 95.4\% (62/65) by SPR. Among the 9 MALDI-TOFMSnegative but SPR-positive samples, sequencing results revealed that they were all non-HR-HPV, which were nontargets of MALDI-TOFMS. However, 5 non-HR-HPV samples as assessed by SPR and sequencing had positive results by MALDI-TOFMS, indicating a cross-reaction of MALDI-TOFMS for LR-HPV. Sequencing analysis of the other 3 discordant samples agreed with MALDITOFMS, which also indicates a cross-reaction of SPR. In conclusion, there was no significant difference between MALDI-TOFMS and SPR regarding cross-reaction.

Furthermore, we used MALDI-TOFMS and SPR to identify the types of 50 cervical cytology samples which had been diagnosed by The Bethesda System. They included 40 cases of NILM, 3 cases of ASC, 5 cases of LSIL, 1 case of HSIL and 1 case of SCC. For the 50 cervical
Table 2. Concordance between MALDI-TOFMS and SPR in HPV genotyping of 79 positive cervical samples

\begin{tabular}{lccl}
\hline $\begin{array}{l}\text { Concordance } \\
\text { type No. }\end{array}$ & $\begin{array}{l}\text { Number of } \\
\text { samples }\end{array}$ & $\begin{array}{l}\text { Concordance } \\
\text { type No. }\end{array}$ & $\begin{array}{l}\text { Number of } \\
\text { samples }\end{array}$ \\
\hline $\begin{array}{l}\text { Single infections } \\
16\end{array}$ & 17 & $\begin{array}{c}\text { multiple infections } \\
16+45\end{array}$ & 1 \\
18 & 4 & $16+52$ & 1 \\
31 & 2 & $16+33$ & 1 \\
33 & 9 & $16+58$ & 1 \\
35 & 2 & $18+58$ & 1 \\
45 & 1 & $31+56$ & 1 \\
51 & 1 & $33+45$ & 1 \\
52 & 2 & $45+56$ & 1 \\
56 & 2 & & \\
58 & 10 & & \\
59 & 1 & & \\
66 & 2 & & \\
68 & 1 & & \\
\hline Subtotal & 54 & & \\
\hline
\end{tabular}


Table 3. Case analysis of discordance between MALDI-TOFMS and SPR for 79 positive cervical samples

\begin{tabular}{clrr}
\hline $\begin{array}{l}\text { Case } \\
\text { No. }\end{array}$ & HPV types & & \\
\cline { 2 - 4 } & MALDI-TOFMS & SPR & sequencing \\
\hline 1 & negative & 53 & 53 \\
2 & negative & 11 & 11 \\
3 & negative & 42 & 42 \\
4 & negative & 6 & 6 \\
5 & negative & 81 & 81 \\
6 & negative & 44 & 44 \\
7 & negative & 70 & 70 \\
8 & negative & 6 & 6 \\
9 & negative & 6 & 6 \\
Cross-reaction of MALDI-TOFMS & & \\
10 & 18 & 11 & 11 \\
11 & 51 & 40 & 40 \\
12 & 56 & 54 & 54 \\
13 & 31 & 6 & 6 \\
14 & 52 & 43 & 43 \\
Cross-reaction of SPR & & \\
15 & 16 & 35 & 16 \\
16 & 16 & 39 & 16 \\
17 & 18 & 45 & 18 \\
\hline
\end{tabular}

samples, the HPV positivity was 32\% (16/50) and 28\% $(14 / 50)$ by MALDI-TOFMS and SPR, respectively. For the 40 NILM samples, the HPV positivity by MALDI-TOFMS and SPR was 20 and $12.5 \%$ (8/40 and 5/40), and $66.7 \%$ (2/3) for ASC, 80 and $100 \%$ (4/5 and 5/5) for LSIL, both $100 \%$ (1/1) for HSIL, and both $100 \%$ (1/1) for SCC, respectively (table 4).

Among the 50 cervical samples, the number of concordant positive and negative samples was 11 (all HR-HPV) and 31, respectively. The concordance between both tests was $84 \%(42 / 50)$, with a $\kappa$ value of 0.660 . For analysis of discordant results, the 8 samples were retested by sequencing to ascertain a consensus result (defined as 2 out of 3 positive or negative HPV test results). Of the 3 samples that were negative by MALDI-TOFMS but positive by SPR, 2 samples were determined to be other than HRHPV types by SPR and sequencing, and 1 sample was determined only by SPR, as shown in table 5 . Among the 5 MALDI-TOFMS-positive but SPR-negative samples, 4 samples were not successfully sequenced because of the lower limit of sequencing. So there were $12 \mathrm{HR}-\mathrm{HPV}$-positive, 2 LR-HPV-positive and 36 negative samples in the 50 cervical samples assessed by the 3 methods.

We summarize that 12 positive samples and 32 negative samples were ascertained by MALDI-TOFMS. The
Table 4. HPV prevalence observed by MALDI-TOFMS and SPR in 50 cervical cytology samples

\begin{tabular}{lcccccc}
\hline & $\begin{array}{l}\text { NILM } \\
(\mathrm{n}=40)\end{array}$ & $\begin{array}{l}\text { ASC } \\
(\mathrm{n}=3)\end{array}$ & $\begin{array}{l}\text { LSIL } \\
(\mathrm{n}=5)\end{array}$ & $\begin{array}{l}\text { HSIL } \\
(\mathrm{n}=1)\end{array}$ & $\begin{array}{l}\text { SCC } \\
(\mathrm{n}=1)\end{array}$ & $\begin{array}{l}\text { Total } \\
(\mathrm{n}=50)\end{array}$ \\
\hline MALDI-TOFMS+ & 8 & 2 & 4 & 1 & 1 & 16 \\
MALDI-TOFMS- & 32 & 1 & 1 & 0 & 0 & 34 \\
Prevalence, \% & 20 & 66.7 & 80 & 100 & 100 & \\
SPR+ & 5 & 2 & 5 & 1 & 1 & 14 \\
SPR- & 35 & 1 & 0 & 0 & 0 & 36 \\
Prevalence, \% & 12.5 & 66.7 & 100 & 100 & 100 & \\
\hline
\end{tabular}

Table 5. Discordant HR-HPV detection by MALDI-TOFMS and SPR in 50 cervical cytology samples (genotyping by sequencing)

\begin{tabular}{|c|c|c|c|c|}
\hline \multirow{2}{*}{$\begin{array}{l}\text { Case } \\
\text { No. }\end{array}$} & \multirow{2}{*}{$\begin{array}{l}\text { Cytology } \\
\text { group }\end{array}$} & \multicolumn{3}{|l|}{ HPV type } \\
\hline & & MALDI-TOFMS & SPR & sequencing \\
\hline 1 & NILM & 52 & negative & negative \\
\hline 2 & NILM & 52 & negative & negative \\
\hline 3 & NILM & 66 & negative & negative \\
\hline 4 & NILM & 31 & negative & negative \\
\hline 5 & NILM & 58 & negative & 58 \\
\hline 6 & NILM & negative & 54 & negative \\
\hline 7 & NILM & negative & 81 & 81 \\
\hline 8 & LSIL & negative & 81 & 81 \\
\hline
\end{tabular}

HR-HPV genotyping accuracy by MALDI-TOFMS was $100 \%(12 / 12)$. There were 13 positive samples and 35 negative samples by SPR. The HR-HPV genotyping accuracy by SPR was $91.7 \%$ (11/12). Among 129 cervical samples, the HR-HPV genotyping accuracy was $100 \%$ (77/77) by MALDI-TOFMS and $94.8 \%$ (73/77) by SPR, respectively. The results indicate that MALDI-TOFMS is as sensitive to HR-HPV as SPR (no significant difference, $\mathrm{p}>0.05$ ).

Sensitivity and Specificity of MALDI-TOFMS and SPR To appreciate the sensitivity and specificity of MALDI-TOFMS and SPR, the results obtained by both tests were compared to consensus HR-HPV results. As shown in table 6 , the sensitivity of MALDI-TOFMS (88.2\%; $82 / 93)$ was similar to that of SPR $(98.9 \%$; $92 / 93)$, as was their specificity (32/36 and 35/36, respectively), in 129 cervical samples. When compared to cytology diagnosis, the sensitivity of both tests was similar in the 3 categories of cytology: ASC, HSIL and SCC. On the other hand, MALDI-TOFMS and SPR were able to successfully identify viral DNA present in HSIL and SCC samples. 
Table 6. Comparison of MALDI-TOFMS and SPR with consensus HR-HPV results in 129 cervical samples

\begin{tabular}{|c|c|c|c|c|c|}
\hline & \multicolumn{2}{|c|}{ Consensus HPV results, $\mathrm{n}$} & \multirow{2}{*}{$\begin{array}{l}\text { Sensitivity } \\
\%\end{array}$} & \multirow{2}{*}{$\begin{array}{l}\text { Specificity } \\
\%\end{array}$} & \multirow[t]{2}{*}{$\kappa$} \\
\hline & + & - & & & \\
\hline MALDI-TOFMS+ & 82 & 4 & $88.2(82 / 93)$ & $88.9(32 / 36)$ & 0.727 \\
\hline MALDI-TOFMS- & 11 & 32 & & & \\
\hline SPR+ & 92 & 1 & $98.9(92 / 93)$ & $97.2(35 / 36)$ & 0.961 \\
\hline SPR- & 1 & 35 & & & \\
\hline
\end{tabular}

Values in parentheses denote numbers.

\section{Discussion}

Some authors have suggested that HPV genotyping could be useful for stratifying the risk for women of developing a precancerous cervical lesion and may be a useful adjunct to cervical cancer screening programs [22]. The aim of the present study was to compare the performance of MALDI-TOFMS to SPR in HR-HPV genotyping, with the purpose of studying the clinical application of MALDI-TOFMS. We also used the sequencing method to resolve discordant results. We collected 1,600 abnormal cervical samples from different A-grade hospitals in China. We found that the HPV positivity was about $53 \%$. According to preliminary statistics, the prevalence of HPV infection was as follows: $33.6 \%$ for HPV16, $12.9 \%$ for HPV 58, 12.4\% for HPV33, 9.6\% for HPV52, 4.3\% for HPV66, 4.1\% for HPV18, 2.6\% for HPV53, 2.5\% for HPV31, 2.4\% for HPV56, 6.7\% for HPV6, 6.5\% for HPV11 and $0.1-2 \%$ for the other HR-HPV and LR-HPV. For comparing the genotyping technologies, we paid more attention to the diversity of HPV types than to the number of samples. Although we selected only $79 \mathrm{HPV}$-positive samples, they covered $24 \mathrm{HPV}$ types which are targeted by SPR. Thus, the 79 samples represent the diversity of HPV types found in the 1,600 samples. We also collected another 50 cervical samples. The total 129 cervical samples were used to evaluate the performance of MALDITOFMS and SPR in genotyping HR-HPV.

Several recent studies also evaluated the performance of MALDI-TOFMS in HPV genotyping. Hong et al. [23] developed a MALDI-TOFMS-based method for HPV genotyping combined with PCR amplification and type IIS restriction which could identify $74 \mathrm{HPV}$ genotypes in 4-5 h. Patel et al. [12] compared HC2 with MALDITOFMS and suggested that the MALDI-TOFMS assay performs at least as well as HC2 in HPV detection while offering the additional, unique advantages of type-spe-

Comparison of MALDI-TOFMS and SPR

for Genotyping of High-Risk HPV cific identification and quantitation. Söderlund-Strand et al. [24] obtained results concordant between MALDITOFMS and reverse dot blot hybridization, with $\kappa=$ 0.945 , in all test samples. In our study, the agreement in HR-HPV genotyping between MALDI-TOFMS and SPR indicates highly comparable outcomes. However, the HRHPV genotyping accuracy was higher by MALDI-TOFMS than by SPR. Among the 129 cervical samples, the HRHPV genotyping accuracy was $100 \%$ (77/77) by MALDITOFMS and $94.8 \%(73 / 77)$ by SPR. The sensitivity $(88.2 \%$; $82 / 93)$ and specificity $(88.9 \% ; 32 / 36)$ of MALDI-TOFMS were similar to those of SPR $(98.9 \%, 92 / 93$, and $97.2 \%$, $35 / 36$, respectively). But in the present study, the number of cervical samples was likely too small to draw definite conclusions. On the other hand, MALDI-TOFMS had a very slightly higher accuracy in genotyping (77/77) than SPR (73/77). Once again, analysis of a larger series of samples would be necessary to confirm these observations. It is of interest to note that the discordant results were observed for LR-HPV samples. This could be explained by probable cross-reactivity of the MALDI-TOFMS assay.

In the present study, we evaluated the MALDI-TOFMS method to detect HR-HPV and compared this assay to SPR and sequencing assays. All the three assays involve PCR amplification by using the MY09/MY11 or GP6/GP5 primers to amplify the consensus region of the HPV L1 gene. The MALDI-TOFMS assay and SPR method amplify a 150-bp region by using GP6/GP5 primers, and sequencing amplifies a 450-bp region by using MY09/ MY11 primers. Some sample genotyping differed among the three methods, owing to the different spectra of the three methods: MALDI-TOFMS targeted 14 HR-HPV, SPR 16 HR-HPV and 8 LR-HPV, and sequencing has the ability to detect all known HPV types as well as potentially unknown types. However, a number of cervical samples genotyped positive by MALDI-TOFMS but negative by SPR or sequencing; this could reflect the higher 
technical sensitivity of MALDI-TOFMS compared to SPR or sequencing.

In summary, our results show that the performance of the MALDI-TOFMS assay in genotyping HR-HPV was identical to that of SPR. The MALDI-TOFMS assay is also able to provide more HPV type information than an estimation of HR-HPV types. Another advantage of MALDI-TOFMS is the high-throughput detection of clinical samples, providing high-quality data. The laboratory procedure for MALDI-TOFMS is also easily adaptable to a 48- or 96-well plate format and readily amenable to automation, making it suitable for large-scale detection in follow-up or primary screening.

\section{Acknowledgments}

This study was supported by the Fund for Major Infectious Disease Diagnostic Products for Quality Evaluation of an Integrated Technology Platform, China (2009ZX10004-805).

\section{References}

$\checkmark 1$ Kjaer S, Høgdall E, Frederiksen K, Munk C, van den Brule A, Svare E, Meijer C, Lorincz A, Iftner T: The absolute risk of cervical abnormalities in high-risk human papillomavirus-positive, cytologically normal women over a 10 -year period. Cancer Res 2006;66: 10630-10636.

-2 Khan MJ, Castle PE, Lorincz AT, Wacholder S, Sherman M, Scott DR, Rush BB, Glass AG, Schiffman M: The elevated 10-year risk of cervical precancer and cancer in women with human papillomavirus (HPV) type 16 or 18 and the possible utility of type-specific $\mathrm{HPV}$ testing in clinical practice. J Natl Cancer Inst 2005;97:1072-1079.

$>3$ Muñoz N, Bosch FX, de Sanjosé S, Herrero R, Castellsagué X, Shah KV, Snijders PJ, Meijer CJ: Epidemiologic classification of human papillomavirus types associated with cervical cancer. N Engl J Med 2003;348:518-527.

-4 Sankaranarayanan R, Nene BM, Shastri SS, Jayant K, Muwonge R, Budukh AM, Hingmire S, Malvi SG, Thorat R, Kothari A, Chinoy R, Kelkar R, Kane S, Desai S, Keskar VR, Rajeshwarkar R, Panse N, Dinshaw KA: HPV screening for cervical cancer in rural India. N Engl J Med 2009;360:1385-1394.

$\checkmark 5$ Walboomers JM, Jacobs MV, Manos MM, Bosch FX, Kummer JA, Shah KV, Snijders PJ, Peto J, Meijer CJ, Muñoz N: Human papillomavirus is a necessary cause of invasive cervical cancer worldwide. J Pathol 1999 189:12-19.

-6 Gontijo RC, Derchain SF, Roteli-Martins C, Bragança JF, Sarian LO, Morais SS, Maeda MY, Longatto-Filho A, Syrjänen KJ: Human papillomavirus (HPV) infections as risk factors for cytological and histological abnormalities in baseline PAP smear-negative women followed-up for 2 years in the LAMS study. Eur J Obstet Gynecol Reprod Biol 2007;133:239-246.

7 Burd EM: Human papillomavirus and cervical cancer. Clin Microbiol Rev 2003;16:1-17.

$\checkmark 8$ Castle PE, Solomon D, Schiffman M, Wheeler CM: Human papillomavirus type 16 infections and 2-year absolute risk of cervical precancer in women with equivocal or mild cytologic abnormalities. J Natl Cancer Inst 2005;97:1066-1071.

$\checkmark 9$ Trottier H, Mahmud S, Costa MC, Sobrinho JP, Duarte-Franco E, Rohan TE, Ferenczy A, Villa LL, Franco EL: Human papillomavirus infections with multiple types and risk of cervical neoplasia. Cancer Epidemiol Biomarkers Prev 2006;15:1274-1280.

10 Agarossi A, Ferrazzi E, Parazzini F, Perno CF, Ghisoni L: Prevalence and type distribution of high-risk human papillomavirus infection in women undergoing voluntary cervical cancer screening in Italy. J Med Virol 2009;81:529-535.

-11 Clavel C, Masure M, Putaud I, Thomas K, Bory JP, Gabriel R, Quereux C, Birembaut P. Hybrid capture II, a new sensitive test for human papillomavirus detection: comparison with hybrid capture I and PCR results in cervical lesions. J Clin Pathol 1998;51:737-740.

12 Patel DA, Shih YJ, Newton DW, Michael CW, Oeth PA, Kane MD, Opipari AW, Ruffin MT 4th, Kalikin LM, Kurnit DM: Development and evaluation of a PCR and mass spectroscopy (PCR-MS)-based method for quantitative, type-specific detection of human papillomavirus. J Virol Methods 2009;160:78-84.

13 Johnson LR, Starkey CR, Palmer J, Taylor J, Stout S, Holt S, Hendren R, Bock B, Waibel E, Tyree G, Miller GC: A comparison of two methods to determine the presence of highrisk HPV cervical infections. Am J Clin Pathol 2008;130:401-408.

14 Schutzbank TE, Jarvis C, Kahmann N, Lopez K, Weimer M, Yount A: Detection of highrisk papillomavirus DNA with commercial invader-technology-based analyte-specific reagents following automated extraction of DNA from cervical brushings in ThinPrep media. J Clin Microbiol 2007;45:4067-4069.

15 Seme K, Fujs K, Kocjan BJ, Poljak M: Resolving repeatedly borderline results of Hybrid Capture 2 HPV DNA Test using polymerase chain reaction and genotyping. J Virol Methods 2006;134:252-256.

16 Poljak M, Marin IJ, Seme K, Vince A: Hybrid Capture II HPV Test detects at least 15 human papillomavirus genotypes not included in its current high-risk probe cocktail. J Clin Virol 2002;25(suppl 3):S89-S97.

17 Bittar F, Ouchenane Z, Smati F, Raoult D, Rolain JM: MALDI-TOF-MS for rapid detection of staphylococcal Panton-Valentine leukocidin. Int J Antimicrob Agents 2009;34: 467-470.

$\checkmark 18$ Hong SP, Kim NK, Hwang SG, Chung HJ, Kim S, Han JH, Kim HT, Rim KS, Kang MS, Yoo W, Kim SO: Detection of hepatitis B virus YMDD variants using mass spectrometric analysis of oligonucleotide fragments. J Hepatol 2004;40:837-844.

-19 Kai E, Ikebukuro K, Hoshina S, Watanabe $\mathrm{H}$, Karube I: Detection of PCR products of Escherichia coli O157:H7 in human stool samples using surface plasmon resonance (SPR). FEMS Immunol Med Microbiol 2000; 29:283-288.

20 Maesawa C, Inaba T, Sato H, Iijima S, Ishida K, Terashima M, Sato R, Suzuki M, Yashima A, Ogasawara S, Oikawa H, Sato N, Saito K, Masuda T: A rapid biosensor chip assay for measuring of telomerase activity using surface plasmon resonance. Nucleic Acids Res 2003;31:E4

21 Castle PE, Schiffman M, Gravitt PE, Kendall H, Fishman S, Dong H, Hildesheim A, Herrero $\mathrm{R}$, Bratti MC, Sherman ME, Lorincz A, Schussler JE, Burk RD: Comparisons of HPV DNA detection by MY09/11 PCR methods. J Med Virol 2002;68:417-423.

22 Dalstein V, Merlin S, Bali C, Saunier M, Dachez R, Ronsin C: Analytical evaluation of the PapilloCheck test, a new commercial DNA chip for detection and genotyping of human papillomavirus. J Virol Methods 2009; 156:77-83.

-23 Hong SP, Shin SK, Lee EH, Kim EO, Ji SI, Chung HJ, Park SN, Yoo W, Folk WR, Kim SO: High-resolution human papillomavirus genotyping by MALDI-TOF mass spectrometry. Nat Protoc 2008;3:1476-1484.

24 Söderlund-Strand A, Dillner J, Carlson J: High-throughput genotyping of oncogenic human papilloma viruses with MALDI-TOF mass spectrometry. Clin Chem 2008;54:8692. 